\title{
New Optical Solitons of the Longitudinal Wave Equation in a Magnetoelectro-Elastic Circular Rod
}

\author{
K. Hosseini ${ }^{a}$, P. Mayeli ${ }^{b}$, E. Yazdani Bejarbaneh ${ }^{c}$ And Qin Zhou ${ }^{d, *}$ \\ ${ }^{a}$ Department of Mathematics, Rasht Branch, Islamic Azad University, Rasht, Iran \\ ${ }^{b}$ Young Researchers and Elite Club, Lahijan Branch, Islamic Azad University, Lahijan, Iran \\ ${ }^{c}$ Young Researchers and Elite Club, Qazvin Branch, Islamic Azad University, Qazvin, Iran \\ ${ }^{d}$ School of Electronics and Information Engineering, Wuhan Donghu University, Wuhan 430212, PR China \\ (Received June 17, 2017; in final form November 6, 2017) \\ This paper studies a nonlinear partial differential equation known as the nonlinear longitudinal wave equation \\ which describes the propagation of optical solitons in a magnetoelectro-elastic circular rod. Mentioned task is \\ performed by solving this nonlinear equation using the well-designed modified Kudryashov method, producing \\ a series of new optical solitons. The capability of the modified Kudryashov method in handling the nonlinear \\ longitudinal wave equation in a magnetoelectro-elastic circular rod is confirmed.
}

DOI: 10.12693/APhysPolA.133.20

PACS/topics: nonlinear longitudinal wave equation, magneto-electro-elastic circular rod, modified Kudryashov method, new optical solitons

\section{Introduction}

Essentially, partial differential equations (PDEs) are of paramount importance among researchers due to its proven capability of modelling a broad range of problems associated with different research areas, such as electronics, mechanics, optics, and plasma physics. Nowadays, by developing a specific transformation, a partial differential equation can be converted into an ordinary differential equation. This conversion causes the produced ordinary differential equation to be solved readily by means of a group of powerful approaches, such as functional variable method [1-3], first integral method [4-7], exp-function method [8-10], modified trial equation method [11-13], the Kudryashov methods [14-19] and so on. The modified Kudryashov method is considered as one of the most robust techniques which has been developed for solving nonlinear differential equations. Numerous studies reveal super performance of this method in handling nonlinear differential equations. For instance, Hosseini and Ansari [20] solved exactly the time-fractional Boussinesq equations using the modified Kudryashov method. Hosseini et al. [21] indicated the suitability of the modified Kudryashov method in solving the nonlinear Tzitzéica type equations in nonlinear optics. Zayed and Alurrfi [22] reported the results of a research in which the modified Kudryashov method was adopted to produce a number of exact solutions for nonlinear seventh-order SawadaKotera-Ito equation, the nonlinear seventh-order KaupKupershmidt equation and the nonlinear seventh-order Lax equation. For more research papers, see [23-42]. This paper illustrates the effectiveness of the modified Kudryashov method in finding a number of new optical solitons for the nonlinear longitudinal wave equation in

*corresponding author; e-mail: qinzhou@whu.edu.cn a magnetoelectro-elastic circular rod [43]

$$
\frac{\partial^{2} u}{\partial t^{2}}-r^{2} \frac{\partial^{2} u}{\partial x^{2}}=\frac{\partial^{2}}{\partial x^{2}}\left(\frac{1}{2} r^{2} u^{2}+n \frac{\partial^{2} u}{\partial t^{2}}\right)
$$

where $r$ is the linear longitudinal wave velocity for a magnetoelectro-elastic circular rod and $n$ is the dispersion parameter, both depending on the material properties as well as the geometry of the rod [43]. This equation was already solved via different methods. For example Baskonus et al. [43] employed the modified $\exp (-\Omega(\xi))$ expansion function method to obtain new analytical solutions of the nonlinear longitudinal wave equation in a magnetoelectro-elastic circular rod. Younis and Ali [44] utilized the ansatz method to extract the bright, dark and singular solitons of the NLWE in a magnetoelectroelastic circular rod. In a similar study, Yang and Xu [45] adopted the ansatz method to construct the 1-soliton solution and peaked solitary wave solutions of the nonlinear longitudinal wave equation in a magnetoelectroelastic circular rod. Xue and coworkers [46] applied the Jacobi elliptic function expansion method for the exact solution of the NLWE in a magnetoelectro-elastic circular rod, and Zhou [47] solved the nonlinear longitudinal wave equation in a magnetoelectro-elastic circular rod via $\left(G^{\prime} / G\right)$-expansion method and traveling wave hypothesis. The rest of this study is structured according to the following sections. Section 2 provides the basic details of the modified Kudryashov method. Section 3 indicates how the longitudinal wave equation in a magnetoelectroelastic circular rod is solved based on the mentioned method to produce a series of new optical solitons. Finally, the last part of the paper is devoted to presenting the conclusion.

\section{Modified Kudryashov method}

Suppose that a nonlinear PDE in two independent variables $x$ and $t$ is demonstrated as 


$$
F\left(u, \frac{\partial u}{\partial t}, \frac{\partial u}{\partial x}, \frac{\partial^{2} u}{\partial t^{2}}, \frac{\partial^{2} u}{\partial x^{2}}, \ldots\right)=0,
$$

where $F$ is a polynomial of $u(x, t)$ and its partial derivatives. Under the wave transformation

$$
u(x, t)=f(\xi), \xi=k x-l t,
$$

Eq. (2) can be reduced to a nonlinear ordinary differential equation in the form

$$
G\left(f, f^{\prime}, f^{\prime \prime}, \ldots\right)=0
$$

where prime denotes derivation with respect to $\xi$. Assume that Eq. (4) has a truncated series solution as follows:

$$
f(\xi)=\sum_{i=0}^{m} a_{i} Q^{i}(\xi)
$$

where $a_{i}, i=0,1, \ldots, m$ are constants to be determined later such that $a_{m} \neq 0$, and $Q=Q(\xi)$ is the solution of the following nonlinear differential equation:

$$
\frac{\mathrm{d} Q}{\mathrm{~d} \xi}=Q(\xi)(Q(\xi)-1) \ln (a) .
$$

It is easy to check that the function $Q(\xi)$ has the form

$$
Q(\xi)=\frac{1}{1+d a^{\xi}}, a \neq 1,
$$

in which $d$ is an arbitrary constant. To find the positive integer $m$, we need to balance the highest order derivative term and the highest order nonlinear term in Eq. (4). By inserting Eq. (5) in Eq. (4), we obtain

$$
c_{0}+c_{1} Q(\xi)+\cdots+c_{\kappa} Q^{\kappa}(\xi)=0
$$

Setting all coefficients in (8) to zero results in a set of nonlinear algebraic equations as

$$
c_{0}=0, c_{1}=0, \ldots, c_{\kappa}=0 .
$$

Solving the system (9) using the symbolic computation package finally generates new exact solutions for the nonlinear PDE (2).

Note 1. The first two derivatives of $f(\xi)$, when $m=2$ are as below

$$
\begin{aligned}
& f^{\prime}(\xi)=a_{1} Q(\xi)(Q(\xi)-1) \ln (a) \\
& \quad+2 a_{2} Q^{2}(\xi)(Q(\xi)-1) \ln (a), \\
& f^{\prime \prime}(\xi)=a_{1} Q(\xi)(Q(\xi)-1)^{2} \ln (a)^{2}+a_{1} Q^{2}(\xi) \\
& \quad \times(Q(\xi)-1) \ln (a)^{2}+4 a_{2} Q^{2}(\xi)(Q(\xi)-1)^{2} \ln (a)^{2} \\
& \quad+2 a_{2} Q^{3}(\xi)(Q(\xi)-1) \ln (a)^{2} .
\end{aligned}
$$

\section{NLWE in a magnetoelectro-elastic circular} rod and its new optical solitons

Using the wave transformation (3) and some mathematical operations, the longitudinal wave equation in a magnetoelectro-elastic circular rod changes into a nonlinear ordinary differential equation as follows:

$$
n l^{2} k^{2} f^{\prime \prime}-\left(l^{2}-k^{2} r^{2}\right) f+\frac{1}{2} k^{2} r^{2} f^{2}=0 .
$$

Balancing $f^{\prime \prime}$ and $f^{2}$ in Eq. (12) gives $m=2$. Therefore, the truncated series (5) becomes

$$
f(\xi)=a_{0}+a_{1} Q(\xi)+a_{2} Q^{2}(\xi) .
$$

By inserting Eq. (13) and Eq. (11) in Eq. (12) and equat- ing the coefficient of each power of $Q(\varepsilon)$ to zero, we will get a system of nonlinear algebraic equations as

$$
\begin{aligned}
& \frac{1}{2} k^{2} r^{2} a_{0}^{2}+k^{2} r^{2} a_{0}-l^{2} a_{0}=0, \\
& l^{2} k^{2} n a_{1} \ln (a)^{2}+k^{2} r^{2} a_{0} a_{1}+k^{2} r^{2} a_{1}-l^{2} a_{1}=0, \\
& -3 l^{2} k^{2} n a_{1} \ln (a)^{2}+4 l^{2} k^{2} n a_{2} \ln (a)^{2}+k^{2} r^{2} a_{0} a_{2} \\
& \quad+\frac{1}{2} k^{2} r^{2} a_{1}^{2}+k^{2} r^{2} a_{2}-l^{2} a_{2}=0, \\
& 2 l^{2} k^{2} n a_{1} \ln (a)^{2}-10 l^{2} k^{2} n a_{2} \ln (a)^{2} \\
& \quad+k^{2} r^{2} a_{1} a_{2}=0, \\
& 6 l^{2} k^{2} n a_{2} \ln (a)^{2}+\frac{1}{2} k^{2} r^{2} a_{2}^{2}=0 .
\end{aligned}
$$

Solving system of nonlinear algebraic equations yields different cases.

\section{Case 1}

$$
a_{0}=0, a_{1}=-a_{2}=\frac{12 l^{2} n \ln (a)^{2}}{r^{2}}, k=\frac{ \pm 1}{\sqrt{n \ln (a)^{2}+\frac{r^{2}}{l^{2}}}},
$$

thus new optical solitons may be presented as follows:

$$
u_{1,2}(x, t)=\frac{12 l^{2} n \ln (a)^{2} d a^{(k x-l t)}}{r^{2}\left(1+d a^{(k x-l t)}\right)^{2}} .
$$

Figure 1 demonstrates three-dimensional graphs of the first new soliton solution for different values of $a$.

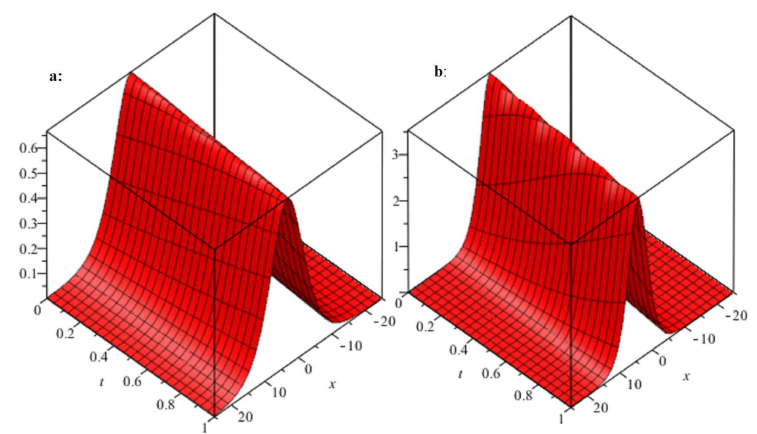

Fig. 1. a: The 3D graph of $u_{1}(x, t)$ when $n=2, r=3$, $l=1, d=1$, and $a=e$; b: the $3 \mathrm{D}$ graph of $u_{1}(x, t)$ when $n=2, r=3, l=1, d=1$, and $a=10$.

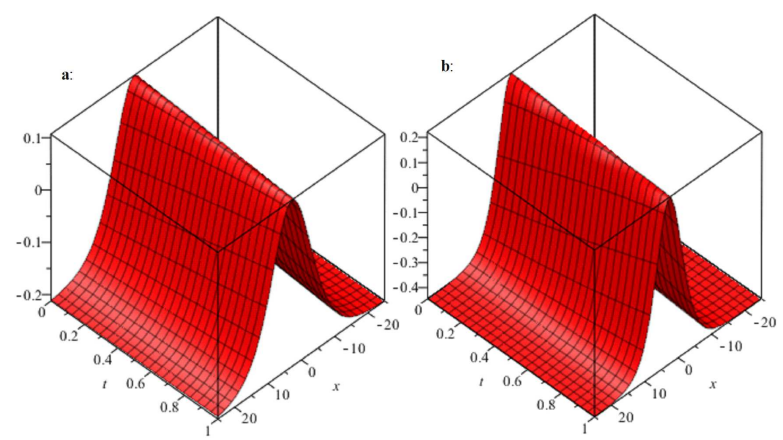

Fig. 2. a: The 3D graph of $u_{3}(x, t)$ when $n=2, r=3$, $l=1, d=1$, and $a=0.5 ; \mathrm{b}$ : the $3 \mathrm{D}$ graph of $u_{3}(x, t)$ when $n=2, r=3, l=1, d=1$, and $a=e$. 


\section{Case 2}

$$
\begin{aligned}
& a_{0}=-\frac{2 l^{2} n \ln (a)^{2}}{r^{2}},-a_{1}=a_{2}=6 a_{0}, \\
& k=\frac{ \pm 1}{\sqrt{-n \ln (a)^{2}+\frac{r^{2}}{l^{2}}}},
\end{aligned}
$$

therefore, new optical solitons may be presented as

$$
u_{3,4}(x, t)=u_{1,2}(x, t)-\frac{2 l^{2} n \ln (a)^{2}}{r^{2}} .
$$

Figure 2 illustrates three-dimensional graphs of the third new soliton solution for different values of $a$.

\section{Conclusion}

This paper explored a nonlinear longitudinal wave equation, illustrating the propagation of optical solitons in magnetoelectro-elastic circular rod. A well-designed technique called the modified Kudryashov method was successfully utilized to extract a number of new optical solitons. The results consisting of new exact solutions show an acceptable level of performance of modified Kudryashov method in handling the model complexity.

\section{Acknowledgments}

This work was supported by National Natural Science Foundation of China under the grant number 11705130.

\section{References}

[1] W. Liu, K. Chen, Pramana J. Phys. 81, 377 (2013).

[2] W. Djoudi, A. Zerarka, 9621 Optik 127, (2016).

[3] A. Nazarzadeh, M. Eslami, M. Mirzazadeh, Pramana J. Phys. 81, 225 (2013).

[4] K. Hosseini, R. Ansari, P. Gholamin, J. Math. Anal. Appl. 387, 807 (2012).

[5] H. Jafari, A. Sooraki, C.M. Khalique, Optik 124, 3929 (2013).

[6] M. Mirzazadeh, A. Biswas, Optik 125, 5467 (2014).

[7] K. Hosseini, P. Gholamin, Diff. Equ. Dyn. Sys. 23, 317 (2015).

[8] X.H. Wu, J.H. He, Chaos Solitons Fractals 38, 903 (2008).

[9] J.H. He, X.H. Wu, Chaos Solitons Fractals 30, 700 (2006).

[10] O. Guner, H. Atik, Optik 127, 10076 (2016).

[11] H. Bulut, H.M. Baskonus, Y. Pandir, Abstr. Appl. Anal. 2013, 636802 (2013).

[12] M. Odabasi, E. Misirli, Math. Meth. Appl. Sci., mma.3533 (2015).

[13] Y.A. Tandogan, N. Bildik, AIP Conf. Proc. 1738 , 290018 (2016).

[14] N.A. Kudryashov, Commun. Nonlin. Sci. Numer. Simul. 17, 2248 (2012).

[15] K. Hosseini, Z. Ayati, Nonlin. Sci. Lett. A 7, 58 (2016).

[16] Z. Ayati, K. Hosseini, M. Mirzazadeh, Nonlinear Eng. 6, 25 (2017).

[17] K. Hosseini, A. Bekir, R. Ansari, Optik 132, 203 (2017).
[18] K. Hosseini, P. Mayeli, R. Ansari, Optik 130, 737 (2017).

[19] S. Saha Ray, Chin. Phys. B 25, 040204 (2016).

[20] K. Hosseini, R. Ansari, Waves Random Complex Media 27, 628 (2017).

[21] K. Hosseini, A. Bekir, M. Kaplan, J. Mod. Opt. 64, 1688 (2017).

[22] E.M.E. Zayed, K.A.E. Alurrfi, World J. Modelling Simul. 11, 308 (2015).

[23] S.M. Ege, E. Misirli, Adv. Diff. Equ. 2014, 135 (2014).

[24] M. Younis, S.T.R. Rizvi, Optik 126, 5812 (2015).

[25] Q. Zhou, M. Ekici, A. Sonmezoglu, M. Mirzazadeh, Optik 127, 6277 (2016).

[26] K. Hosseini, A. Bekir, R. Ansari, J. Quant. Electron. 49, 131 (2017).

[27] Q. Zhou, M. Mirzazadeh, M. Ekici, A. Sonmezoglu, Nonlin. Dyn. 86, 623 (2016)

[28] A.H. Arnous, M.Z. Ullah, S.P. Moshokoa, Q. Zhou, H. Triki, M. Mirzazadeh, A. Biswas, Nonlin. Dyn. 88, 1891 (2017).

[29] M. Ekici, M. Mirzazadeh, M. Eslami, Nonlin. Dyn. 84, 669 (2016)

[30] H. Tariq, G. Akram, Nonlin. Dyn. 88, 581 (2017).

[31] A. Sonmezoglu, M. Yao, M. Ekici, M. Mirzazadeh, Q. Zhou, Nonlin. Dyn. 88, 595 (2017).

[32] B. Muatjetjeja, O.P. Porogo, Nonlinear Dyn. 89, 443 (2017).

[33] H. Triki, T. Ak, M. Ekici, A. Sonmezoglu, M. Mirzazadeh, A.H. Kara, T. Aydemir, Nonlin. Dyn. 89, 501 (2017).

[34] L.D. Moleleki, B. Muatjetjeja, A.R. Adem, Nonlin. Dyn. 87, 2187 (2017)

[35] R. Shi, Z. Song, T. Feng, G. Wang, X. Wang, Nonlin. Dyn. 88, 255 (2017).

[36] A.M. Wazwaz, Nonlin. Dyn. 83, 591 (2016).

[37] A.M. Wazwaz, J. Ocean Eng. Sci. 1, 181 (2016).

[38] H. Triki, A.M. Wazwaz, Phys. Lett. A 380, 1260 (2016).

[39] H.H. Zhao, X.J. Zhao, H.Q. Hao, Appl. Math. Lett. 61, 8 (2016).

[40] H.Q. Hao, R. Guo, J.W. Zhang, Nonlin. Dyn. 88, 1615 (2017)

[41] R. Guo, H.Q. Hao, Commun. Nonlin. Sci. Numer. Simul. 18, 2426 (2013)

[42] X.J. Zhao, R. Guo, H.Q. Hao, Appl. Math. Lett. 75 114 (2018).

[43] H.M. Baskonus, H. Bulut, A. Atangana, Smart Mater. Struct. 25, 035022 (2016)

[44] M. Younis, S. Ali, Waves Random Complex Media 25, 549 (2015).

[45] S. Yang, T. Xu, Nonlin. Dyn. 87, 2735 (2017).

[46] C.X. Xue, E. Pan, S.Y. Zhang, Smart Mater. Struct. 20, 105010 (2011).

[47] Q. Zhou, Nonlin. Dyn. 83, 1403 (2016). 